\title{
DIRICHLET PRODUCT AND THE MULTIPLE DIRICHLET SERIES OVER FUNCTION FIELDS
}

\author{
YOSHINORI HAMAHATA \\ Okayama University of Science, Japan
}

\begin{abstract}
We define the Dirichlet product for multiple arithmetic functions over function fields and consider the ring of the multiple Dirichlet series over function fields. We apply our results to absolutely convergent multiple Dirichlet series and obtain some zero-free regions for them.
\end{abstract}

\section{INTRODUCTION}

A function $f: \mathbb{N} \rightarrow \mathbb{C}$ is called an arithmetic function. For arithmetic functions $f$ and $g$, the Dirichlet product $f * g$ is defined as

$$
(f * g)(n)=\sum_{d \mid n} f(d) g\left(\frac{n}{d}\right) .
$$

Using this product, we can obtain many results in number theory (see Apostol $[3])$. For an arithmetic function $f: \mathbb{N} \rightarrow \mathbb{C}$, the Dirichlet series $L(s ; f)$ is defined as

$$
L(s ; f):=\sum_{n=1}^{\infty} \frac{f(n)}{n^{s}}
$$

which includes the Riemann zeta function $\zeta(s)=\sum_{n=1}^{\infty} n^{-s}$ and the Dirichlet $L$-function $L(s, \chi)=\sum_{n=1}^{\infty} \chi(n) n^{-s}$ for the Dirichlet character $\chi$. To know the location of the zeros of $L(s ; f)$, its zero-free region is often studied. For example, the Riemann zeta function $\zeta(s)$ is absolutely convergent when $\operatorname{Re}(s)>1$, and has no zeros in this region. The multiple Dirichlet series,

2020 Mathematics Subject Classification. 11R58, 11A25, 11M32, 11M41.

Key words and phrases. Arithmetic function, Dirichlet product, Dirichlet series, zeta function, function field. 
which is a multiple variable generalization of the Dirichlet series, is defined as

$$
\sum_{n_{1}=1}^{\infty} \cdots \sum_{n_{k}=1}^{\infty} \frac{f\left(n_{1}, \ldots, n_{k}\right)}{n_{1}^{s_{1}} \cdots n_{k}^{s_{k}}}
$$

where $f: \mathbb{N}^{k} \rightarrow \mathbb{C}$ is a multiple arithmetic function. As examples of such multiple Dirichlet series, we have the Euler-Zagier multiple zeta function $\zeta_{E Z, k}\left(s_{1}, \ldots, s_{k}\right)$, the multiple zeta star function $\zeta_{k}^{\star}\left(s_{1}, \ldots, s_{k}\right)$, and the Mordell-Tornheim multiple zeta function $\zeta_{M T, k}\left(s_{1}, \ldots, s_{k}\right)$ :

$$
\begin{aligned}
\zeta_{E Z, k}\left(s_{1}, \ldots, s_{k}\right) & =\sum_{0<n_{1}<\cdots<n_{k}} \frac{1}{n_{1}^{s_{1}} \cdots n_{k}^{s_{k}}}, \\
\zeta_{k}^{\star}\left(s_{1}, \ldots, s_{k}\right) & =\sum_{0<n_{1} \leq \cdots \leq n_{k}} \frac{1}{n_{1}^{s_{1}} \cdots n_{k}^{s_{k}}}, \\
\zeta_{M T, k}\left(s_{1}, \ldots, s_{k} ; s_{k+1}\right) & =\sum_{n_{1}=1}^{\infty} \cdots \sum_{n_{k}=1}^{\infty} \frac{1}{n_{1}^{s_{1}} \cdots n_{k}^{s_{k}}\left(n_{1}+\cdots+n_{k}\right)^{s_{k+1}}} .
\end{aligned}
$$

It is known that $\zeta_{E Z, k}\left(s_{1}, \ldots, s_{k}\right)$ and $\zeta_{k}^{\star}\left(s_{1}, \ldots, s_{k}\right)$ are absolutely convergent in

$$
\operatorname{Re}\left(s_{i}+\cdots+s_{k}\right)>k-i+1 \quad(i=1, \ldots, k),
$$

and that $\zeta_{M T, k}\left(s_{1}, \ldots, s_{k} ; s_{k+1}\right)$ is absolutely convergent in

$$
\operatorname{Re}\left(s_{i}\right)>1 \quad(i=1, \ldots, k), \quad \operatorname{Re}\left(s_{k+1}\right)>0 .
$$

For details, we refer the reader to Matsumoto, $[7,8]$. To study the multiple Dirichlet series, Onozuka in [9] investigated the Dirichlet product on the set of multiple arithmetic functions. As an application, he provided a result regarding a zero-free region for the multiple Dirichlet series.

The set $\mathcal{D}\left[\left[s_{1}, \ldots, s_{k}\right]\right]$ of all multiple formal Dirichlet series becomes a ring using ordinary addition and product operations. Onozuka in [9] proved that $\mathcal{D}\left[\left[s_{1}, \ldots, s_{k}\right]\right]$ is a unique factorization domain.

There is an analogy between number fields and function fields in one variable over the finite field $\mathbb{F}_{q}$. The theory of complex-valued zeta functions exists in function fields, as in $[10,12]$. To study the Dirichlet series over function fields, we first introduce the Dirichlet product of multiple arithmetic functions over function fields. We subsequently consider the ring of the multiple formal Dirichlet series. We apply our results to absolutely convergent multiple Dirichlet series to obtain some of their zero-free regions.

The remainder of this paper is organized as follows. In Section 2, we define the Dirichlet product of the multiple arithmetic functions over function fields. Using this, we show that the set of all multiple arithmetic functions becomes a unique factorization domain. We subsequently define multiple formal Dirichlet series over function fields and show that these series form a unique factorization domain. In Section 3, using the results from the previous 
sections, we investigate the zero-free regions for absolutely convergent multiple Dirichlet series.

Notation. $\mathbb{N}_{0}$ is the set of non-negative integers, $\mathbb{R}_{>0}$ is the set of positive real numbers and $f(\mathbf{a}) \ll g(\mathbf{a})$ means $|f(\mathbf{a})|=O(g(\mathbf{a}))$.

\section{Multiple arithmetic Functions}

In this section, we introduce multiple arithmetic functions over function fields and investigate their Dirichlet products. For the Dirichlet product in the classical case, we refer the reader to $[1,2,3,4,5,9,11,13]$.

Henceforth, let $\mathbb{F}_{q}$ be the finite field with $q$ elements, where $q$ is a power of the prime number $p$. Let $A=\mathbb{F}_{q}[T]$ and let $A_{+}$be the set of all monic polynomials in $A$. Let $k$ be a positive integer. We use bold letters to express the elements of $A_{+}^{k}$. For example, we denote $\left(a_{1}, \ldots, a_{k}\right),(1, \ldots, 1) \in A_{+}^{k}$ as $\mathbf{a}, \mathbf{1}$, respectively. For $\mathbf{a}=\left(a_{1}, \ldots, a_{k}\right), \mathbf{b}=\left(b_{1}, \ldots, b_{k}\right) \in A_{+}^{k}$, we write $\mathbf{a}+\mathbf{b}=\left(a_{1}+b_{1}, \ldots, a_{k}+b_{k}\right), \mathbf{a} \cdot \mathbf{b}=\left(a_{1} b_{1}, \ldots, a_{k} b_{k}\right)$.

2.1. An ordering of $A_{+}^{k}$. We set

$$
\mathcal{P}_{k}:=\left\{\left(1, \ldots, 1, P_{j}, 1, \ldots, 1\right) \in A_{+}^{k} \mid P \text { is irreducible, } 1 \leq j \leq k\right\} .
$$

An element $\mathbf{P} \in \mathcal{P}_{k}$ is called a multiple prime. In particular, an element $\mathbf{P} \in \mathcal{P}_{k}$ is called a prime when $k=1$. Because $\mathcal{P}_{k}$ is countable, there exists a bijection $c: \mathbb{N} \rightarrow \mathcal{P}_{k}$. For $j \in \mathbb{N}$, let $\mathbf{P}_{j}=c(j)$. We see easily that any element $\mathbf{M} \in A_{+}^{k}$ can be written as the product of finite multiple primes.

Let

$$
|a|= \begin{cases}q^{\operatorname{deg} a} & \text { if } a \neq 0 \\ 0 & \text { if } a=0\end{cases}
$$

for $a \in A$.

Let $\mathbb{N}_{0}^{(\infty)}$ be the set of all sequences of the non-negative integers with finite supports. Namely,

$$
\mathbb{N}_{0}^{(\infty)}=\left\{\left(\alpha_{1}, \ldots, \alpha_{n}, \ldots\right) \mid \alpha_{i} \in \mathbb{N}_{0}, \alpha_{i}=0 \text { for almost all } i\right\} .
$$

Thus, there exists a bijection $\psi: A_{+}^{k} \rightarrow \mathbb{N}_{0}^{(\infty)}$ defined as

$$
\psi\left(\prod_{i=1}^{\infty} \mathbf{P}_{i}^{\alpha_{i}}\right)=\left(\alpha_{1}, \ldots, \alpha_{n}, \ldots\right) .
$$

When $\mathbf{P}_{i}=(1, \ldots, 1, P, 1, \ldots, 1)$, let $c_{i}=\log |P|$. We define

$$
L: \mathbb{N}_{0}^{(\infty)} \rightarrow \mathbb{R}, \quad\left(\alpha_{1}, \ldots, \alpha_{n}, \ldots\right) \mapsto \sum_{i=1}^{\infty} c_{i} \alpha_{i} .
$$


Using $L$, we define an ordering of $A_{+}^{k}$ as follows: Take $\mathbf{a}=\left(a_{1}, \ldots, a_{k}\right), \mathbf{b}=$ $\left(b_{1}, \ldots, b_{k}\right) \in A_{+}^{k}$ such that

$$
\mathbf{a}=\prod_{i=1}^{\infty} \mathbf{P}_{i}^{\alpha_{i}} \neq \mathbf{b}=\prod_{i=1}^{\infty} \mathbf{P}_{i}^{\beta_{i}}
$$

When

$$
(L \circ \psi)(\mathbf{a})=\log \left|a_{1} \cdots a_{k}\right|<(L \circ \psi)(\mathbf{b})=\log \left|b_{1} \cdots b_{k}\right|,
$$

let $\mathbf{a}<_{L} \mathbf{b}$. When $(L \circ \psi)(\mathbf{a})=(L \circ \psi)(\mathbf{b})$, let $\mathbf{a}<_{L} \mathbf{b}$ if $\alpha_{1}=\beta_{1}, \ldots, \alpha_{i-1}=$ $\beta_{i-1}$, and $\alpha_{i}<\beta_{i}$ for some $i$. We write $\min _{L}$ for the minimum with respect to $L$.

2.2. The multiple Dirichlet product. We call $f: A_{+}^{k} \rightarrow \mathbb{C}$ a multiple $(k$ tuple) arithmetic function. Let

$$
\Omega_{k}=\left\{f \mid f: A_{+}^{k} \rightarrow \mathbb{C}\right\}
$$

be the set of multiple arithmetic functions. For $f, g \in \Omega_{k}$, the sum $f+g$ is defined as $(f+g)(\mathbf{a})=f(\mathbf{a})+g(\mathbf{b})$.

Definition 2.1. For $f, g \in \Omega_{k}$, the multiple Dirichlet product $f * g$ is defined as

$$
(f * g)(\mathbf{a})=\sum_{\substack{\mathbf{b} \cdot \mathbf{c}=\mathbf{a} \\ \mathbf{b}, \mathbf{c} \in A_{+}^{k}}} f(\mathbf{b}) g(\mathbf{c})
$$

This product is called the Dirichlet product when $k=1$.

To discuss the algebraic structure of $\Omega_{k}$, we define the norm $N: \Omega_{k} \rightarrow \mathbb{N}_{0}$ as

$$
N(f)=\left\{\begin{array}{cc}
0 & \text { if } f=0 \\
\min _{\mathbf{a} \in A_{+}^{k}}\left\{\left|a_{1}\right| \cdots\left|a_{k}\right| \mid f(\mathbf{a}) \neq 0\right\} & \text { if } f \neq 0
\end{array}\right.
$$

for $f \in \Omega_{k}$. The following result holds for the norm:

Proposition 2.2. (i) For $f \in \Omega_{k}, N(f)=1$ if and only if $f(\mathbf{1}) \neq 0$.

(ii) For $f, g \in \Omega_{k}, N(f * g)=N(f) N(g)$.

Proof. (i) is easy. (ii) If $f=0$ or $g=0$, then $f * g=0$. Hence, the equality is valid. Assume that $f \neq 0$ and $g \neq 0$. We take $\mathbf{a} \in A_{+}$such that $\left|a_{1}\right| \cdots\left|a_{k}\right|<N(f) N(g)$. If $\mathbf{a}=\mathbf{b} \cdot \mathbf{c}$ for $\mathbf{b}, \mathbf{c} \in A_{+}^{k}$, then $\left|b_{1}\right| \cdots\left|b_{k}\right|<N(f)$ or $\left|c_{1}\right| \cdots\left|c_{k}\right|<N(g)$. Hence, $(f * g)(\mathbf{a})=0$. Let

$$
\begin{aligned}
\mathbf{L} & =\left(L_{1}, \ldots, L_{k}\right)=\min _{L}\left\{\mathbf{b} \in A_{+}^{k} \mid f(\mathbf{b}) \neq 0\right\}, \\
\mathbf{M} & =\left(M_{1}, \ldots, M_{k}\right)=\min _{L}\left\{\mathbf{c} \in A_{+}^{k} \mid g(\mathbf{c}) \neq 0\right\} .
\end{aligned}
$$


Thus, $N(f)=\left|L_{1}\right| \cdots\left|L_{k}\right|, N(g)=\left|M_{1}\right| \cdots\left|M_{k}\right|$. We take $\mathbf{b}, \mathbf{c} \in A_{+}^{k}$ such that $\mathbf{b} \cdot \mathbf{c}=\mathbf{M} \cdot \mathbf{L}$. If $\mathbf{b}<_{L} \mathbf{L}$, then $f(\mathbf{b})=0$. If $\mathbf{b}>_{L} \mathbf{L}$, then $\mathbf{c}<_{L} \mathbf{M}$, hence $g(\mathbf{c})=0$. Therefore, we have

$$
(f * g)(\mathbf{L} \cdot \mathbf{M})=\sum_{\mathbf{b} \cdot \mathbf{c}=\mathbf{L} \cdot \mathbf{M}} f(\mathbf{b}) g(\mathbf{c})=f(\mathbf{L}) g(\mathbf{M}) \neq 0,
$$

which yields $N(f * g)=\left|L_{1}\right| \cdots\left|L_{k}\right|\left|M_{1}\right| \cdots\left|M_{k}\right|=N(f) N(g)$.

2.3. The ring of arithmetic functions. Using + and $*$ defined above, $\Omega_{k}$ becomes a ring as follows:

Proposition 2.3. (i) $\Omega_{k}$ is an integral domain with the identity function $I: \Omega_{k} \rightarrow \mathbb{C}$, which is defined as

$$
I(\mathbf{a})= \begin{cases}1 & \text { if } \mathbf{a}=\mathbf{1} \\ 0 & \text { if } \mathbf{a} \neq \mathbf{1}\end{cases}
$$

(ii) The group of units of $\Omega_{k}$ is

$$
U_{k}:=\left\{f \in \Omega_{k} \mid f(\mathbf{1}) \neq 0\right\} .
$$

Proof. (i) We see easily that $\Omega_{k}$ is a commutative ring with identity I. We take $f, g \in \Omega_{k}$ with $f \neq 0, g \neq 0$. According to Proposition 2.2, $N(f * g) \neq 0$, which yields $f * g \neq 0$. Hence, $\Omega_{k}$ is an integral domain.

(ii) For $f \in U_{k}$, we define $f^{-1} \in \Omega_{k}$ as

$$
f^{-1}(\mathbf{a})=\left\{\begin{array}{cc}
\frac{1}{f(\mathbf{1})} & \text { if } \mathbf{a}=\mathbf{1}, \\
-\frac{1}{f(\mathbf{1})} \sum_{\substack{\mathbf{b} \cdot \mathbf{c}=\mathbf{a} \\
\mathbf{c} \neq \mathbf{a}}} f(\mathbf{b}) f^{-1}(\mathbf{c}) & \text { if } \mathbf{a} \neq \mathbf{1} .
\end{array}\right.
$$

Then, it holds that $f * f^{-1}=I$. Hence, $f$ is a unit of $\Omega_{k}$. Conversely, if $f$ is a unit of $\Omega_{k}$, then there exists $g \in \Omega_{k}$ such that $f * g=I$. We have $N(f)=1$ because $N(f) N(g)=N(f * g)=1$. Hence, $f \in U_{k}$ by Proposition 2.2.

The ring $\Omega_{k}$ has the following properties.

THEOREM 2.4. (i) $\Omega_{k}$ is a unique factorization domain.

(ii) $\Omega_{k}$ is a local ring.

(iii) $\Omega_{k}$ is not a Noetherian ring.

Proof. (i) For each $j \in \mathbb{N}$, the map $\alpha_{j}: A_{+}^{k} \rightarrow \mathbb{N}_{0}$ is defined as

$$
\mathbf{M}=\mathbf{P}_{1}^{\alpha_{1}(\mathbf{M})} \mathbf{P}_{2}^{\alpha_{2}(\mathbf{M})} \cdots .
$$

It is easy to see that $\alpha_{j}(\mathbf{a} \cdot \mathbf{b})=\alpha_{j}(\mathbf{a})+\alpha_{j}(\mathbf{b})$ for $\mathbf{a}, \mathbf{b} \in A_{+}^{k}$. Let $x_{1}, x_{2}, \ldots, x_{n}, \ldots$ be distinct indeterminates, and let $\mathbb{C}_{\omega}:=\mathbb{C}\left\{x_{1}, x_{2}, \ldots, x_{n}, \ldots\right\}$ 
be the ring of the formal power series in $x_{1}, x_{2}, \ldots, x_{n}, \ldots$ We define a map $R: \Omega_{k} \rightarrow \mathbb{C}_{\omega}$ as

$$
R(f)=\sum_{\mathbf{M} \in A_{+}^{k}} f(\mathbf{M}) x_{1}^{\alpha_{1}(\mathbf{M})} x_{2}^{\alpha_{2}(\mathbf{M})} \cdots .
$$

We first prove that $R$ is a ring isomorphism. It is easy to see that $R(I)=1$. Let $f, g \in \Omega_{k}$. It is easy see that $R(f+g)=R(f)+R(g)$. We have

$$
\begin{aligned}
R(f * g) & =\sum_{\mathbf{M} \in A_{+}^{k} \mathbf{a} \cdot \mathbf{b}=\mathbf{M}} f(\mathbf{a}) g(\mathbf{b}) x_{1}^{\alpha_{1}(\mathbf{M})} x_{2}^{\alpha_{2}(\mathbf{M})} \ldots \\
& =\left(\sum_{\mathbf{a} \in A_{+}^{k}} f(\mathbf{a}) x_{1}^{\alpha_{1}(\mathbf{a})} x_{2}^{\alpha_{2}(\mathbf{a})} \ldots\right)\left(\sum_{\mathbf{b} \in A_{+}^{k}} g(\mathbf{b}) x_{1}^{\alpha_{1}(\mathbf{b})} x_{2}^{\alpha_{2}(\mathbf{b})} \ldots\right) \\
& =R(f) R(g) .
\end{aligned}
$$

Next, we see easily that $R$ is injective. Finally, for $E \in \mathbb{C}_{\omega}$, we can find $f_{E} \in \Omega_{k}$ such that $R\left(f_{E}\right)=E$. Hence, $R$ is surjective. According to [4], $\mathbb{C}_{\omega}$ is a unique factorization domain. This finishes the proof of (i).

(ii) Let $\mathfrak{m}=\left\{f \in \Omega_{k} \mid f(\mathbf{1})=0\right\}$. We see easily that $\mathfrak{m}$ is an ideal of $\Omega_{k}$. Hence, $\Omega_{k}$ is a local ring with maximal ideal $\mathfrak{m}$ because $\Omega_{k} \backslash U_{k}=\mathfrak{m}$.

(iii) In the ring $\mathbb{C}_{\omega}$, the chain of ideals

$$
\left(x_{1}\right) \subset\left(x_{1}, x_{2}\right) \subset \cdots \subset\left(x_{1}, x_{2}, \ldots, x_{n}\right) \subset \cdots
$$

does not become stationary. Hence, $\Omega_{k}$ is not Noetherian.

Because $\Omega_{k} \cong \mathbb{C}_{\omega}$, we have the following result.

COROLlary 2.5. $\Omega_{k}$ is isomorphic to $\Omega_{l}$ for all positive integers $k$ and $l$.

2.4. The ring of the multiple formal Dirichlet series. Let

$$
\mathcal{D}\left[\left[s_{1}, \ldots, s_{k}\right]\right]:=\left\{\sum_{\mathbf{a} \in A_{+}^{k}} \frac{f(\mathbf{a})}{\left|a_{1}\right|^{s_{1}} \cdots\left|a_{k}\right|^{s_{k}}} \mid f \in \Omega_{k}\right\},
$$

whose elements are called the multiple formal Dirichlet series. For $f, g \in \Omega_{k}$, we understand that

$$
\sum_{\mathbf{a} \in A_{+}^{k}} f(\mathbf{a})\left|a_{1}\right|^{-s_{1}} \cdots\left|a_{k}\right|^{-s_{k}}=\sum_{\mathbf{a} \in A_{+}^{k}} g(\mathbf{a})\left|a_{1}\right|^{-s_{1}} \cdots\left|a_{k}\right|^{-s_{k}}
$$

if and only if $f=g$. For $F \in \mathcal{D}\left[\left[s_{1}, \ldots, s_{k}\right]\right]$, we use $f$ to denote the arithmetic function defined as

$$
F\left(s_{1}, \ldots, s_{k}\right)=F\left(s_{1}, \ldots, s_{k} ; f\right):=\sum_{\mathbf{a} \in A_{+}^{k}} \frac{f(\mathbf{a})}{\left|a_{1}\right|^{s_{1}} \cdots\left|a_{k}\right|^{s_{k}}} .
$$


ExAmple 2.6. $F(s ; f)=\sum_{a \in A_{+}} f(a)|a|^{-s}$ is simply called the Dirichlet series associated to $f$ for $f \in \Omega_{1}$ when $k=1$. In particular,

$$
\zeta_{A}(s):=\sum_{a \in A_{+}} \frac{1}{|a|^{s}}
$$

is called the zeta function for $A$. This function is absolutely convergent when $\operatorname{Re}(s)>1$, and can be written as

$$
\zeta_{A}(s)=\frac{1}{1-q^{1-s}}=\prod_{\substack{P \in A_{+} \\ \text {irreducible }}}\left(1-\frac{1}{|P|^{s}}\right)^{-1} .
$$

Let $M \in A$ with $\operatorname{deg} M>0$. A Dirichlet character modulo $M$ is a function $\chi: A \rightarrow \mathbb{C}$ such that

(i) $\chi(a+b M)=\chi(a)$ for $a, b \in A$.

(ii) $\chi(a b)=\chi(a) \chi(b)$ for $a, b \in A$.

(iii) $\chi(a) \neq 0$ if and only if $\operatorname{gcd}(a, M)=1$.

The Dirichlet L-function for $\chi$ is defined as

$$
L(s, \chi):=\sum_{a \in A_{+}} \frac{\chi(a)}{|a|^{s}}
$$

which is absolutely convergent when $\operatorname{Re}(s)>1$, and can be written as

$$
L(s, \chi)=\prod_{\substack{P \in A_{+} \\ \text {irreducible }}}\left(1-\frac{\chi(P)}{|P|^{s}}\right)^{-1} .
$$

For the further details, we refer the reader to Rosen, [10].

ExAMPLE 2.7. (i) For the arithmetic function $u^{\star} \in \Omega_{k}$ defined as

$$
u^{\star}(\mathbf{a})= \begin{cases}1 & \text { if }\left|a_{1}\right| \leq \cdots \leq\left|a_{k}\right| \\ 0 & \text { otherwise }\end{cases}
$$

the multiple zeta star function is defined as

$$
\zeta_{k}^{\star}\left(s_{1}, \ldots, s_{k}\right):=F\left(s_{1}, \ldots, s_{k} ; u^{\star}\right)=\sum_{\left|a_{1}\right| \leq \cdots \leq\left|a_{k}\right|} \frac{1}{\left|a_{1}\right|^{s_{1} \cdots\left|a_{k}\right|^{s_{k}}} .}
$$

(ii) For the arithmetic function $u_{E Z} \in \Omega_{k}$ defined as

$$
u_{E Z}(\mathbf{a})= \begin{cases}1 & \text { if }\left|a_{1}\right|<\cdots<\left|a_{k}\right|, \\ 0 & \text { otherwise, }\end{cases}
$$

the Euler-Zagier multiple zeta function is defined as

$$
\zeta_{E Z, k}\left(s_{1}, \ldots, s_{k}\right):=F\left(s_{1}, \ldots, s_{k} ; u_{E Z}\right)=\sum_{\left|a_{1}\right|<\cdots<\left|a_{k}\right|} \frac{1}{\left|a_{1}\right|^{s_{1} \cdots\left|a_{k}\right|^{s_{k}}}} .
$$


(iii) The Mordell-Tornheim multiple zeta function is defined as

$$
\begin{aligned}
\zeta_{M T, k}\left(s_{1}, \ldots, s_{k} ; s_{k+1}\right) & :=F\left(s_{1}, \ldots, s_{k+1} ; u_{M T}\right) \\
& =\sum_{\mathbf{a} \in A_{+}^{k}} \frac{1}{\left|a_{1}\right|^{s_{1}} \cdots\left|a_{k}\right|^{s_{k}}\left(\left|a_{1}\right|+\cdots+\left|a_{k}\right|\right)^{s_{k+1}}} .
\end{aligned}
$$

The ring $\mathcal{D}\left[\left[s_{1}, \ldots, s_{k}\right]\right]$ becomes a ring with the addition and product operations, defined as

$$
\begin{aligned}
\sum_{\mathbf{a} \in A_{+}^{k}} \frac{f(\mathbf{a})}{\left|a_{1}\right|^{s_{1} \cdots\left|a_{k}\right|^{s_{k}}}}+\sum_{\mathbf{a} \in A_{+}^{k}} \frac{g(\mathbf{a})}{\left|a_{1}\right|^{s_{1} \cdots\left|a_{k}\right|^{s_{k}}}}=\sum_{\mathbf{a} \in A_{+}^{k}} \frac{(f+g)(\mathbf{a})}{\left|a_{1}\right|^{s_{1} \cdots\left|a_{k}\right|^{s_{k}}}}, \\
\left(\sum_{\mathbf{a} \in A_{+}^{k}} \frac{f(\mathbf{a})}{\left|a_{1}\right|^{s_{1} \cdots\left|a_{k}\right|^{s_{k}}}}\right)\left(\sum_{\mathbf{a} \in A_{+}^{k}} \frac{g(\mathbf{a})}{\left|a_{1}\right|^{s_{1} \ldots\left|a_{k}\right|^{s_{k}}}}\right)=\sum_{\mathbf{a} \in A_{+}^{k}} \frac{(f * g)(\mathbf{a})}{\left|a_{1}\right|^{s_{1} \ldots\left|a_{k}\right|^{s_{k}}}} .
\end{aligned}
$$

The correspondence $f \mapsto \sum_{\mathbf{a} \in A_{+}^{k}} f(\mathbf{a})\left|a_{1}\right|^{-s_{1}} \ldots\left|s_{k}\right|^{-s_{k}}$ induces a ring isomorphism $\Omega_{k} \cong \mathcal{D}\left[\left[s_{1}, \ldots, s_{k}\right]\right]$. According to Theorem 2.4 and Corollary 2.5, we have the two following results.

THEOREM 2.8. (i) $\mathcal{D}\left[\left[s_{1}, \ldots, s_{k}\right]\right]$ is a unique factorization domain.

(ii) $\mathcal{D}\left[\left[s_{1}, \ldots, s_{k}\right]\right]$ is a local ring.

(iii) $\mathcal{D}\left[\left[s_{1}, \ldots, s_{k}\right]\right]$ is not a Noetherian ring.

Corollary 2.9. $\mathcal{D}\left[\left[s_{1}, \ldots, s_{k}\right]\right]$ is isomorphic to $\mathcal{D}\left[\left[s_{1}, \ldots, s_{l}\right]\right]$ for all positive integers $k$ and $l$.

\section{Application}

In this section, we consider the zero-free regions for absolutely convergent multiple Dirichlet series over function fields.

3.1. Regions of absolute convergence. For the zeta functions in Example 2.7, we have the following regions of absolute convergence.

LEMMA 3.1. (i) $\zeta_{k}^{\star}\left(s_{1}, \ldots, s_{k}\right)$ is absolutely convergent in the region

$$
\operatorname{Re}\left(s_{i}+\cdots+s_{k}\right)>k-i+1 \quad(i=1, \ldots, k) .
$$

Moreover, $\zeta_{k}^{\star}\left(s_{1}, \ldots, s_{k}\right)$ can be written as

$$
\zeta_{k}^{\star}\left(s_{1}, \ldots, s_{k}\right)=\frac{1}{\left(1-q^{k-\left(s_{1}+\cdots+s_{k}\right)}\right)\left(1-q^{k-1-\left(s_{2}+\cdots+s_{k}\right)}\right) \cdots\left(1-q^{1-s_{k}}\right)} .
$$

(ii) $\zeta_{E Z, k}\left(s_{1}, \ldots, s_{k}\right)$ is absolutely convergent in the region (3.1). Moreover, $\zeta_{E Z, k}\left(s_{1}, \ldots, s_{k}\right)$ can be written as

$$
\zeta_{E Z, k}\left(s_{1}, \ldots, s_{k}\right)=\frac{q^{\left(1-s_{2}\right)+2\left(1-s_{3}\right)+\cdots+(k-1)\left(1-s_{k}\right)}}{\left(1-q^{k-\left(s_{1}+\cdots+s_{k}\right)}\right)\left(1-q^{k-1-\left(s_{2}+\cdots+s_{k}\right)}\right) \cdots\left(1-q^{1-s_{k}}\right)} .
$$


(iii) $\zeta_{M T, k}\left(s_{1}, \ldots, s_{k}\right)$ is absolutely convergent in the region

$$
\operatorname{Re}\left(s_{i}\right)>1 \quad(i=1, \ldots, k), \quad \operatorname{Re}\left(s_{k+1}\right)>0 .
$$

Proof. (i) See Masri, [6, Theorem 1.1]. (ii) See Thakur, [12, 5.10].

(iii) The following holds in the region (3.3):

$$
\sum_{\mathbf{a} \in A_{+}^{k}}\left|\frac{1}{\left|a_{1}\right|^{s_{1}} \cdots\left|a_{k}\right|^{s_{k}}\left(\left|a_{1}\right|+\cdots+\left|a_{k}\right|\right)^{s_{k+1}}}\right|<\zeta_{A}\left(\operatorname{Re}\left(s_{1}\right)\right) \cdots \zeta_{A}\left(\operatorname{Re}\left(s_{k}\right)\right),
$$

which yields the proof of (iii).

From this lemma, we can see easily the following.

Corollary 3.2. (i) For the Dirichlet characters $\chi_{1}, \ldots, \chi_{k}$, we define the multiple L-star function as

$$
L_{k}^{\star}\left(s_{1}, \ldots, s_{k} ; \chi_{1}, \ldots, \chi_{k}\right):=\sum_{\left|a_{1}\right| \leq \cdots \leq\left|a_{k}\right|} \frac{\chi_{1}\left(a_{1}\right) \cdots \chi_{k}\left(a_{k}\right)}{\left|a_{1}\right|^{s_{1} \cdots\left|a_{k}\right|^{s_{k}}}} .
$$

Then, this L-function is absolutely convergent in the region (3.1).

(ii) For the Dirichlet characters $\chi_{1}, \ldots, \chi_{k}$, we define the Euler-Zagier multiple L-function as

$$
L_{E Z, k}\left(s_{1}, \ldots, s_{k} ; \chi_{1}, \ldots, \chi_{k}\right):=\sum_{\left|a_{1}\right|<\cdots<\left|a_{k}\right|} \frac{\chi_{1}\left(a_{1}\right) \cdots \chi_{k}\left(a_{k}\right)}{\left|a_{1}\right|^{s_{1} \cdots\left|a_{k}\right|^{s_{k}}} .}
$$

Then, this L-function is absolutely convergent in the region (3.1).

3.2. Zero-free regions. To consider the multiple Dirichlet series analogous to the multiple zeta functions in the example above, we introduce the subsets of $\Omega_{k}$ as follows:

$$
\begin{aligned}
\Omega_{k}^{\star} & :=\left\{f \in \Omega_{k} \mid f(\mathbf{a})=0 \text { for a which does not satisfy }\left|a_{1}\right| \leq \cdots \leq\left|a_{k}\right|\right\}, \\
\Omega_{E Z, k} & :=\left\{f \in \Omega_{k} \mid f(\mathbf{a})=0 \text { for a which does not satisfy }\left|a_{1}\right|<\cdots<\left|a_{k}\right|\right\}, \\
\Omega_{M T, k} & :=\left\{f \in \Omega_{k} \mid f(\mathbf{a})=0 \text { for a which satisfies }\left|a_{k}\right|<\left|a_{1}\right|+\cdots+\left|a_{k-1}\right|\right\} .
\end{aligned}
$$

Using + and $*$, these subsets become subrings of $\Omega_{k}$ as follows.

Proposition 3.3. (i) $\Omega_{k}^{\star}$ is a subring of $\Omega_{k}$.

(ii) $\Omega_{E Z, k}$ is a subring of $\Omega_{k}$ such that $\Omega_{E Z, k} \cap U_{k}=\phi$.

(iii) $\Omega_{M T, k}$ is a subring of $\Omega_{k}$ such that $\Omega_{M T, k} \cap U_{k}=\phi$.

Proof. It is easy to see that $\Omega_{k}^{*}, \Omega_{E Z, k}$, and $\Omega_{M T, k}$ are additive subgroups of $\Omega_{k}$.

(i) Let $f, g \in \Omega_{k}^{\star}$. We take $\mathbf{a} \in A_{+}^{k}$ which does not satisfy $\left|a_{1}\right| \leq \cdots \leq$ $\left|a_{k}\right|$. If $\mathbf{b} \cdot \mathbf{c}=\mathbf{a}$ for $\mathbf{b}, \mathbf{c} \in A_{+}^{k}$, then $\left|b_{1}\right| \leq \cdots \leq\left|b_{k}\right|$ or $\left|c_{1}\right| \leq \cdots \leq\left|c_{k}\right|$ does not hold. Hence, $(f * g)(\mathbf{a})=\sum_{\mathbf{b} \cdot \mathbf{c}=\mathbf{a}} f(\mathbf{b}) g(\mathbf{c})=0$, which implies that $f * g \in \Omega_{k}^{\star}$. Because of $I \in \Omega_{k}^{\star} \cap U_{k}, \Omega_{k}^{\star} \cap U_{k} \neq \phi$. 
(ii) The former is proved as was the case for (i). We have $f(\mathbf{1})=0$ for any $f \in \Omega_{E Z, k}$. This implies $\Omega_{E Z, k} \cap U_{k}=\phi$.

(iii) Let $f, g \in \Omega_{M T, k}$, and let $\mathbf{a}, \mathbf{b}, \mathbf{c} \in A_{+}^{k}$ such that $\mathbf{b} \cdot \mathbf{c}=\mathbf{a}$. If $\left|b_{k}\right| \geq\left|b_{1}\right|+\cdots+\left|b_{k-1}\right|$ and $\left|c_{k}\right| \geq\left|c_{1}\right|+\cdots+\left|c_{k-1}\right|$, then

$$
\begin{aligned}
\left(\left|a_{1}\right|+\cdots+\left|a_{k-1}\right|\right)^{2} & \leq\left(\left|b_{1}\right|\left|c_{1}\right|+\cdots+\left|b_{k-1}\right|\left|c_{k-1}\right|\right)^{2} \\
& \leq\left(\left|b_{1}\right|^{2}+\cdots+\left|b_{k-1}\right|^{2}\right)\left(\left|c_{1}\right|^{2}+\cdots+\left|c_{k-1}\right|^{2}\right) \\
& \leq\left(\left|b_{1}\right|+\cdots+\left|b_{k-1}\right|\right)^{2}\left(\left|c_{1}\right|+\cdots+\left|c_{k-1}\right|\right)^{2} \\
& \leq\left|b_{k}\right|^{2}\left|c_{k}\right|^{2}=\left|a_{k}\right|^{2}
\end{aligned}
$$

which yields $\left|a_{k}\right| \geq\left|a_{1}\right|+\cdots+\left|a_{k-1}\right|$. Hence, if $\left|a_{k}\right|<\left|a_{1}\right|+\cdots+\left|a_{k-1}\right|$, then

$$
\left|b_{k}\right|<\left|b_{1}\right|+\cdots+\left|b_{k-1}\right| \text { or }\left|c_{k}\right|<\left|c_{1}\right|+\cdots+\left|c_{k-1}\right| \text {. }
$$

Hence, $(f * g)(\mathbf{a})=\sum_{\mathbf{b} \cdot \mathbf{c}=\mathbf{a}} f(\mathbf{b}) g(\mathbf{c})=0$, which implies $f * g \in \Omega_{M T, k}$. We have $f(\mathbf{1})=0$ for any $f \in \Omega_{M T, k}$. This implies $\Omega_{M T, k} \cap U_{k}=\phi$.

According to Proposition 3.3, we have the following equalities:

$$
\begin{aligned}
& \left(\sum_{\left|a_{1}\right| \leq \cdots \leq\left|a_{k}\right|} \frac{f(\mathbf{a})}{\left|a_{1}\right|^{s_{1}} \cdots\left|a_{k}\right|^{s_{k}}}\right)\left(\sum_{\left|a_{1}\right| \leq \cdots \leq\left|a_{k}\right|} \frac{g(\mathbf{a})}{\left|a_{1}\right|^{s_{1}} \cdots\left|a_{k}\right|^{s_{k}}}\right) \\
& =\sum_{\left|a_{1}\right| \leq \cdots \leq\left|a_{k}\right|} \frac{(f * g)(\mathbf{a})}{\left|a_{1}\right|^{s_{1} \cdots\left|a_{k}\right|^{s_{k}}}} \quad\left(f, g \in \Omega_{k}^{\star}\right), \\
& \left(\sum_{\left|a_{1}\right|<\cdots<\left|a_{k}\right|} \frac{f(\mathbf{a})}{\left|a_{1}\right|^{s_{1}} \cdots\left|a_{k}\right|^{s_{k}}}\right)\left(\sum_{\left|a_{1}\right|<\cdots<\left|a_{k}\right|} \frac{g(\mathbf{a})}{\left|a_{1}\right|^{s_{1}} \cdots\left|a_{k}\right|^{s_{k}}}\right) \\
& =\sum_{\left|a_{1}\right|<\cdots<\left|a_{k}\right|} \frac{(f * g)(\mathbf{a})}{\left|a_{1}\right|^{s_{1}} \cdots\left|a_{k}\right|^{s_{k}}} \quad\left(f, g \in \Omega_{E Z, k}\right), \\
& \left(\sum_{\left|a_{k}\right| \geq\left|a_{1}\right|+\cdots+\left|a_{k}\right|} \frac{f(\mathbf{a})}{\left|a_{1}\right|^{s_{1}} \cdots\left|a_{k}\right|^{s_{k}}}\right)\left(\sum_{\left|a_{k}\right| \geq\left|a_{1}\right|+\cdots+\left|a_{k}\right|} \frac{g(\mathbf{a})}{\left|a_{1}\right|^{s_{1}} \cdots\left|a_{k}\right|^{s_{k}}}\right) \\
& =\sum_{\left|a_{k}\right| \geq\left|a_{1}\right|+\cdots+\left|a_{k}\right|} \frac{(f * g)(\mathbf{a})}{\left|a_{1}\right|^{s_{1} \cdots\left|a_{k}\right|^{s_{k}}}} \quad\left(f, g \in \Omega_{M T, k}\right) .
\end{aligned}
$$

Moreover, for $f \in \Omega_{k}^{\star} \cap U_{k}$,

$$
\left(\sum_{\left|a_{1}\right| \leq \cdots \leq\left|a_{k}\right|} \frac{f(\mathbf{a})}{\left|a_{1}\right|^{s_{1}} \cdots\left|a_{k}\right|^{s_{k}}}\right)^{-1}=\sum_{\left|a_{1}\right| \leq \cdots \leq\left|a_{k}\right|} \frac{f^{-1}(\mathbf{a})}{\left|a_{1}\right|^{s_{1}} \cdots\left|a_{k}\right|^{s_{k}}} .
$$

Corollary 3.4. Assume that $F\left(s_{1}, \ldots, s_{k} ; f\right)$ and $F\left(s_{1}, \ldots, s_{k} ; f^{-1}\right)$ are absolutely convergent on $R \subset \mathbb{C}^{k}$ for $f \in U_{k}$. Then, $F\left(s_{1}, \ldots, s_{k} ; f\right)$ has no zeros on $R$. 
Proof. Using (2.2), for $\left(s_{1}, \ldots, s_{k}\right) \in R$,

$$
F\left(s_{1}, \ldots, s_{k} ; f\right) F\left(s_{1}, \ldots, s_{k} ; f^{-1}\right)=F\left(s_{1}, \ldots, s_{k} ; I\right)=1 .
$$

We need the following lemma to prove Theorem 3.6.

Lemma 3.5. (i) $\quad \sum_{d \mid a}|d|^{\alpha} \leq \zeta_{A}(\alpha)|a|^{\alpha}$ for all $\alpha>1$.

(ii) For every $f \in U_{k}$, there exist $C>0$ and $r_{1}, \ldots, r_{k} \in \mathbb{R}$ such that

$$
|f(\mathbf{a})| \leq C\left|a_{1}\right|^{r_{1}} \cdots\left|a_{k}\right|^{r_{k}}
$$

for all $\mathbf{a} \in A_{+}^{k} \backslash\{\mathbf{1}\}$. For such $f$, choose $\alpha_{j}>1+r_{j}(j=1, \ldots, k)$ such that

$$
\zeta_{A}\left(\alpha_{1}-r_{1}\right) \cdots \zeta_{A}\left(\alpha_{k}-r_{k}\right) \leq 1+\frac{|f(\mathbf{1})|}{C} .
$$

Then, for all $\mathbf{a} \in A_{+}^{k}$,

$$
\left|f^{-1}(\mathbf{a})\right| \leq \frac{\left|a_{1}\right|^{\alpha_{1}} \cdots\left|a_{k}\right|^{\alpha_{k}}}{|f(\mathbf{1})|} .
$$

Proof. (i) We have

$$
\sum_{d \mid a}|d|^{\alpha}=|a|^{\alpha} \sum_{d \mid a}\left|\frac{d}{a}\right|^{\alpha}=|a|^{\alpha} \sum_{d \mid a} \frac{1}{|d|^{\alpha}} \leq \zeta_{A}(\alpha)|a|^{\alpha} .
$$

(ii) We prove the result by induction on $d=\operatorname{deg} a_{1}+\cdots+\operatorname{deg} a_{k}$. If $d=0$, then $\mathbf{a}=\mathbf{1}$, which yields $\left|f^{-1}(\mathbf{1})\right|=|f(\mathbf{1})|^{-1}$. Let $d>0$ and we assume that the theorem holds for $\mathbf{c} \in A_{+}^{k}$ with $\operatorname{deg} c_{1}+\cdots+\operatorname{deg} c_{k}<d$. Then, using (i), we have

$$
\begin{aligned}
&\left|f^{-1}(\mathbf{a})\right| \leq \frac{1}{|f(\mathbf{1})|} \sum_{\substack{\mathbf{b} \cdot \mathbf{c}=\mathbf{a} \\
\mathbf{c} \neq \mathbf{a}}}|f(\mathbf{b})| \cdot\left|f^{-1}(\mathbf{c})\right| \\
& \leq \frac{C}{|f(\mathbf{1})|^{2}} \sum_{\substack{\mathbf{b} \cdot \mathbf{c}=\mathbf{a} \\
\mathbf{c} \neq \mathbf{a}}}\left|b_{1}\right|^{r_{1}}\left|c_{1}\right|^{\alpha_{1}} \ldots\left|b_{k}\right|^{r_{k}}\left|c_{k}\right|^{\alpha_{k}} \\
&=\frac{C}{|f(\mathbf{1})|^{2}} \sum_{\substack{\mathbf{c} \mid \mathbf{a} \\
\mathbf{c} \neq \mathbf{a}}}\left|\frac{a_{1}}{c_{1}}\right|^{r_{1}}\left|c_{1}\right|^{\alpha_{1}} \ldots\left|\frac{a_{k}}{c_{k}}\right|^{r_{k}}\left|c_{k}\right|^{\alpha_{k}} \\
&=\frac{C}{|f(\mathbf{1})|^{2}}\left[\left|a_{1}\right|^{r_{1}} \ldots\left|a_{k}\right|^{r_{k}}\left(\sum_{c_{1} \mid a_{1}}\left|c_{1}\right|^{\alpha_{1}-r_{1}}\right) \cdots\left(\sum_{c_{k} \mid a_{k}}\left|c_{k}\right|^{\alpha_{k}-r_{k}}\right)\right. \\
&\left.-\left|a_{1}\right|^{\alpha_{1}} \ldots\left|a_{k}\right|^{\alpha_{k}}\right]
\end{aligned}
$$




$$
\begin{aligned}
& \leq \frac{C}{|f(\mathbf{1})|^{2}}\left(\zeta_{A}\left(\alpha_{1}-r_{1}\right)\left|a_{1}\right|^{\alpha_{1}} \cdots \zeta_{A}\left(\alpha_{k}-r_{k}\right)\left|a_{k}\right|^{\alpha_{k}}-\left|a_{1}\right|^{\alpha_{1}} \cdots\left|a_{k}\right|^{\alpha_{k}}\right) \\
& \leq \frac{\left|a_{1}\right|^{\alpha_{1}} \cdots\left|a_{k}\right|^{\alpha_{k}}}{|f(\mathbf{1})|}
\end{aligned}
$$

THEOREM 3.6. Given the same notations and assumptions as those used in Lemma 3.5, $F\left(s_{1}, \ldots, s_{k} ; f\right)$ and $F\left(s_{1}, \ldots, s_{k} ; f^{-1}\right)$ have no zeros on

$$
\left\{\left(s_{1}, \ldots, s_{k}\right) \in \mathbb{C}^{k} \mid \operatorname{Re}\left(s_{j}\right)>1+\alpha_{j} \quad(j=1, \ldots, k)\right\} .
$$

Moreover, it holds that

$$
F\left(s_{1}, \ldots, s_{k} ; f\right)^{-1}=F\left(s_{1}, \ldots, s_{k} ; f^{-1}\right)
$$

in this region.

Proof. The multiple Dirichlet series $F\left(s_{1}, \ldots, s_{k} ; f\right)$ is absolutely convergent on $\operatorname{Re}\left(s_{j}\right)>1+r_{j}(j=1, \ldots, k)$ because $f(\mathbf{a}) \ll\left|a_{1}\right|^{r_{1}} \ldots\left|a_{k}\right|^{r_{k}}$. Using Lemma 3.5, $F\left(s_{1}, \ldots, s_{k} ; f^{-1}\right)$ is absolutely convergent on the region (3.4) because $\left|f^{-1}(\mathbf{a})\right| \ll\left|a_{1}\right|^{\alpha_{1}} \cdots\left|a_{k}\right|^{\alpha_{k}}$. The latter part is easy.

Using Lemma 3.1 and Theorem 3.6, we have the following improved zerofree region for $f \in \Omega_{k}^{\star} \cap U_{k}$.

Theorem 3.7. Take $f \in \Omega_{k}^{\star} \cap U_{k}$ satisfying the condition in Lemma 3.5. Then, $F\left(s_{1}, \ldots, s_{k} ; f\right)$ and $F\left(s_{1}, \ldots, s_{k} ; f^{-1}\right)$ do not have zeros on

$\left\{\left(s_{1}, \ldots, s_{k}\right) \in \mathbb{C}^{k} \mid \operatorname{Re}\left(s_{i}+\cdots+s_{k}\right)>k-i+1+\alpha_{i}+\cdots+\alpha_{k} \quad(i=1, \ldots, k)\right\}$.

Proof. Using Lemma 3.1 (i), $F\left(s_{1}, \ldots, s_{k} ; f\right)$ is absolutely convergent in $\operatorname{Re}\left(s_{i}+\cdots+s_{k}\right)>k-i+1+r_{i}+\cdots+r_{k}(i=1, \ldots, k)$ because $|f(\mathbf{a})| \ll$ $\left|a_{1}\right|^{r_{1}} \ldots\left|a_{k}\right|^{r_{k}}$. In the same way, using Lemma 3.1 (i), $F\left(s_{1}, \ldots, s_{k} ; f^{-1}\right)$ is absolutely convergent in $\operatorname{Re}\left(s_{i}+\cdots+s_{k}\right)>k-i+1+\alpha_{i}+\cdots+\alpha_{k}(i=1, \ldots, k)$ because $\left|f^{-1}(\mathbf{a})\right| \ll\left|a_{1}\right|^{\alpha_{1}} \cdots\left|a_{k}\right|^{\alpha_{k}}$, which completes the proof.

Example 3.8. The multiple Dirichlet series $\zeta_{k}^{\star}\left(s_{1}, \ldots, s_{k}\right), L_{k}^{\star}\left(s_{1}, \ldots, s_{k}\right)$, $\zeta_{E Z, k}\left(s_{1}, \ldots, s_{k} ; \chi_{1}, \ldots, \chi_{k}\right)+1$, and $L_{E Z, k}\left(s_{1}, \ldots, s_{k} ; \chi_{1}, \ldots, \chi_{k}\right)+1$ belong to $\Omega_{k}^{\star} \cap U_{k}$. In Lemma 3.5 (ii), we can take $r_{1}=\cdots=r_{k}=0$, and $C=|f(\mathbf{1})|=1$. Using Theorems 3.6 and 3.7, we see that these series do not have zeros in the region

$\left\{\left(s_{1}, \ldots, s_{k}\right) \in \mathbb{C}^{k} \mid \operatorname{Re}\left(s_{i}+\cdots+s_{k}\right)>k-i+1+\alpha_{i}+\cdots+\alpha_{k} \quad(j=1, \ldots, k)\right\}$, for $\alpha_{i}>1(i=1, \ldots, k)$ such that $\zeta_{A}\left(\alpha_{1}\right) \cdots \zeta_{A}\left(\alpha_{k}\right) \leq 2$.

REMARK 3.9. It is possible that the zero-free region in Theorem 3.7 is not the best region. For example, we consider $\zeta_{2}^{\star}\left(s_{1}, s_{2}\right)$. When, $q \geq \sqrt{2} /(\sqrt{2}-1)$, $\zeta_{A}(2)^{2} \leq 2$. Then, using $(3.5), \zeta_{2}^{\star}\left(s_{1}, s_{2}\right)$ has no zeros in

$$
\operatorname{Re}\left(s_{1}+s_{2}\right)>6, \quad \operatorname{Re}\left(s_{2}\right)>3 .
$$


However, using Lemma $3.1, \zeta_{2}^{\star}\left(s_{1}, s_{2}\right)$ is absolutely convergent in

$$
\operatorname{Re}\left(s_{1}+s_{2}\right)>2, \quad \operatorname{Re}\left(s_{2}\right)>1,
$$

and has no zeros in this region.

ACKNOWLEDGEMENTS.

The author would like to thank the referees for the helpful comments that improved this paper.

\title{
REFERENCES
}

[1] E. Alkan, A. Zaharescu and M. Zaki, Arithmetical functions in several variables, Int. J. Number Theory 1 (2005), 383-399.

[2] E. Alkan, A. Zaharescu and M. Zaki, Multidimensional averages and Dirichlet convolution, Manuscripta Math. 123 (2007), 251-267.

[3] T. Apostol, Introduction to analytic number theory, Springer, 1976.

[4] E. Cashwell and C. Everett, The ring of number-theoretic functions, Pacific J. Math. 9 (1959), 975-985.

[5] P. Haukkanen, Derivation of arithmetical functions under the Dirichlet convolution, Int. J. Number Theory 14 (2018), 1257-1264.

[6] R. Masri, Multiple zeta values over global function fields, Proc. Sympos. Pure Math. 75, 2006, 157-175.

[7] K. Matsumoto, On analytic continuation of various multiple zeta-functions, In: Number Theory for the Millennium, Vol. 2, A. K. Peters, 2002, pp. 417-440.

[8] K. Matsumoto, On Mordell-Tornheim and other multiple zeta-functions, In: Proceedings of the Session in Analytic Number Theory and Diophantine Equations, 2003, No. 25, 17pp.

[9] T. Onozuka, The multiple Dirichlet product and the multiple Dirichlet series, Int. J. Number Theory 13 (2017), 2181-2193.

[10] M. Rosen, Number theory in function fields, Springer, 2002.

[11] R. Sivaramakrishnan, Classical theory of arithmetic functions, Marcel Dekker, Inc., New York, 1989.

[12] D. Thakur, Function field arithmetic, World Scientific, River Edge, 2004.

[13] L. Tóth, Multiplicative arithmetic functions of several variables: a survey, In: T. Rassias and P. Pardalos (eds.), Mathematics without boundaries, Springer, 2014, 483514.

\author{
Y. Hamahata \\ Department of Applied Mathematics \\ Okayama University of Science \\ Ridai-cho 1-1 \\ Okayama \\ 700-0005 \\ Japan \\ E-mail: hamahata@xmath.ous.ac.jp
}

Received: 3.5.2019.

Revised: 13.11.2019. 